\title{
Medical Data Mining Using Evolutionary
}

\section{Computation}

\author{
Po Shun Ngan* \\ Man Leung Wong** \\ Wai $\mathrm{Lam}^{+}$ \\ psngan@cse.cuhk.edu.hk \\ mlwong@cse.cuhk.edu.hk \\ wlam@se.cuhk.edu.hk \\ Kwong Sak Leung* \\ Jack C. Y. Cheng ${ }^{++}$ \\ ksleung@cse.cuhk.edu.hk \\ jackcheng@cuhk.edu.hk \\ *Department of Computer Science and Engineering \\ The Chinese University of Hong Kong, Hong Kong \\ ${ }^{* *}$ Department of Computer Studies \\ Lingnan College, Hong Kong \\ +Department of Systems Engineering and Engineering Management \\ The Chinese University of Hong Kong, Hong Kong \\ ${ }^{++}$Department of Orthopaedics and Traumatology \\ The Chinese University of Hong Kong, Hong Kong
}




\begin{abstract}
In this paper, we introduce a system for discovering medical knowledge by learning Bayesian networks and rules. Evolutionary Computation is used as the search algorithm. The Bayesian networks can provide an overall structure of the relationships among the attributes. The rules can capture detailed and interesting patterns in the database. The system is applied to two real-life medical databases. The knowledge discovered provides insights to and allows better understanding of the medical domains.
\end{abstract}

Keywords: Data Mining, Evolutionary Computation, Rule Learning, Bayesian Networks, Grammar Based Genetic Programming 


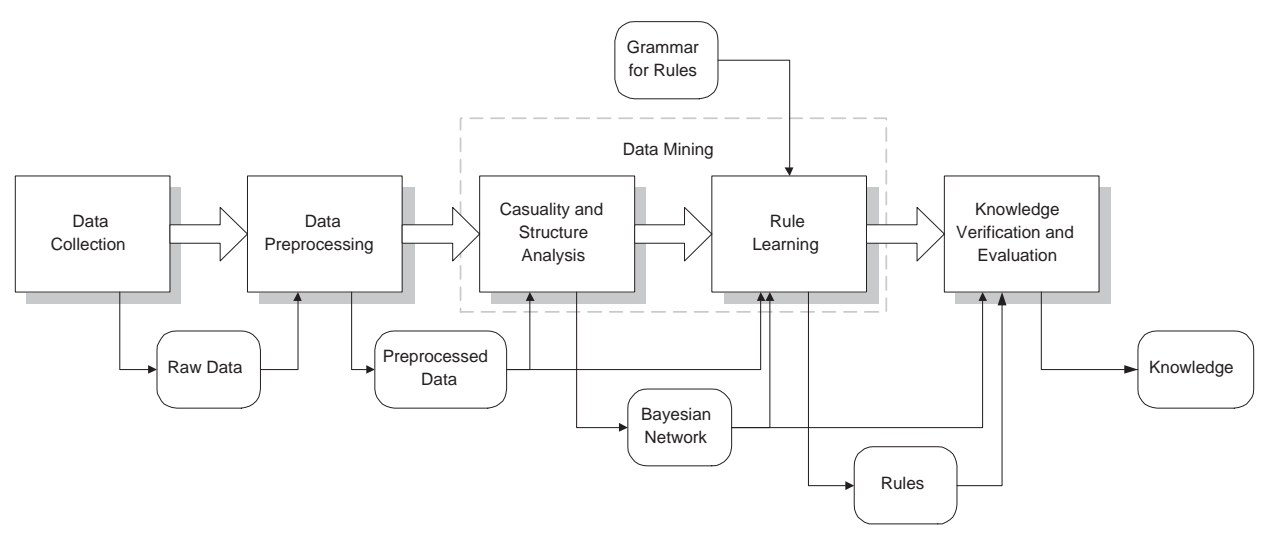

Figure 1: The knowledge discovery process

\section{Introduction}

Data mining aims at discovering novel, interesting and useful knowledge from databases [9]. Conventionally, the data is analyzed manually. Many hidden and potentially useful relationships may not be recognized by the analyst. Nowadays, many organizations are capable of generating and collecting a huge amount of data. This explosive growth of data generated an urgent need for an automated way to extract useful knowledge from the data. With the computerization in hospitals, a huge amount of data is collected. These data provide the basis for learning knowledge. Thus, medical domain is a major area for applying data mining. Through data mining, we can extract interesting knowledge and regularities. The discovered knowledge can finally be applied in the corresponding field to increase the working efficiency and improve the quality of decision making.

We developed a knowledge discovery system to learn knowledge from data. There are five steps in the system (Figure 1). Real-life data are collected in the first step. Then, the data must be preprocessed before analysis can be started. The third and fourth steps induce knowledge from the preprocessed data. The Causality and Structure Analysis step learns the overall relationships between the variables. A Bayesian network is an output 
to represent the knowledge structure. Based on this knowledge, the user can specify the target relationships (s)he wants to know by formulating a grammar. The Rule Learning step learns a set of significant rules from the data. The grammar can guide the format of the rules to be learned. In the fifth step, the learned knowledge is verified and evaluated by the domain experts. The domain experts may discover and correct mistakes in the learned knowledge. On the other hand, the learned knowledge can be used to refine the existing domain knowledge. Finally, the learned Bayesian network is used to perform reasoning under uncertainty, and the induced rules are incorporated into an expert system for decision making.

In this paper, we present the two knowledge learning steps, which are the core of the knowledge discovery system. They both employ Evolutionary Computation as the search algorithms. This paper is organized as follows. Section 2 introduces the backgrounds on Evolutionary Computation, Bayesian network learning, and rule learning. Section 3 describes the approaches for learning Bayesian networks. The rule learning process is delineated in Section 4 and the details of the techniques are given in section 5 . The data mining system has been applied to two real-life medical databases. The results are presented in section 6 and 7 . Section 8 is the conclusion.

\section{Backgrounds}

\subsection{Evolutionary Computation}

The term Evolutionary Computation is used to describe algorithms that simulate the natural evolution to perform function optimization and machine learning. They are based on 
the Darwinian principle of evolution through natural selection. The algorithms maintain a group of individuals to explore the search space. Examples of Evolutionary Computation include Genetic Algorithms (GA) [19, 13], Genetic Programming (GP) [24, 25], Evolutionary Programming (EP) [10, 11] and Evolution Strategy (ES) [34, 35]. GA uses a fixed-length binary bit string as an individual. Three genetic operators are used to search for better individuals. Reproduction operator just copies the individual unchanged to the new generation. Crossover operator exchanges bits between two parents. Mutation operator randomly changes individual bits. GP extends GA by using a tree structure as the individual. EP emphasizes on the behavioral linkage between parents and their offspring. Mutation is the only genetic operator in EP. There is no constraint on the representation in EP. ES emphasizes on the individual, i.e. the phenotype, to be the object to be optimized. A genetic change in the individual is within a narrow band of the mutation step size and the step size has self-adaptations.

Data Mining can be considered as a search problem, which tries to find the most accurate knowledge from all possible hypotheses. Since evolutionary computation is a robust and parallel search algorithm, it can be used in data mining to find interesting knowledge in noisy environment.

\subsection{Bayesian network Learning}

Bayesian network is a formal knowledge representation supported by the well-developed Bayesian probability theory. A Bayesian network captures the conditional probabilities between attributes. It can be used to perform reasoning under uncertainty. A Bayesian network is a directed acyclic graph. Each node represents a domain variable, and each edge 


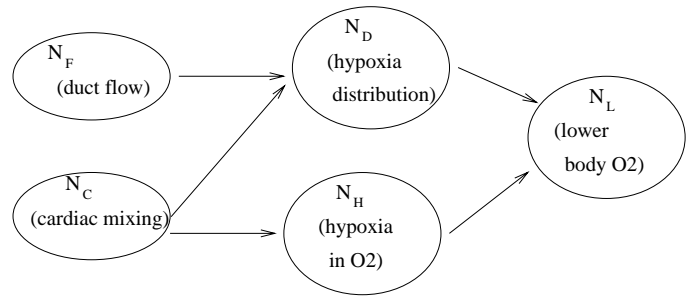

Figure 2: A Bayesian Network Structure in a "Blue" Baby Domain

represents a dependency between two nodes. An edge from node $A$ to node $B$ can represent a causality, with $A$ being the cause and $B$ being the effect. The value of each variable should be discrete. Each node is associated with a set of parameters. Let $N_{i}$ denote a node and $\Pi_{N_{i}}$ denote the set of parents of $N_{i}$. The parameters of $N_{i}$ are conditional probability distributions in the form of $P\left(N_{i} \mid \Pi_{N_{i}}\right)$, with one distribution for each possible instance of $\Pi_{N_{i}}$. Figure 2 is an example Bayesian network structure modeling a medical domain concerned with "blue" baby diagnosis. More details of Bayesian networks can be found in $[4,17,15]$.

The main task of learning Bayesian network from data is to automatically find directed edges between the nodes, such that the network can best describe the causalities. Once the network structure is constructed, the conditional probabilities are calculated based on the data. The problem of Bayesian network learning is computationally intractable [7]. However, Bayesian networks learning can be implemented by imposing limitations and assumptions. For instance, the algorithms of Chow and Liu [5] and Rebane and Pearl [33] can learn networks with tree structures, while the algorithms of Herskovits and Cooper $[18,8]$ and Bouckaert [3] require the variables to have a total ordering. More general algorithms include Heckerman et. al. [16], Spirtes et. al. [39] and Singh and Valtorta [36]. More recently, Larranaga et. al. [30, 29] has proposed algorithms for learning Bayesian 
networks using Genetic Algorithms.

\subsection{Rule Learning}

A rule is a sentence of the form "if antecedents, then consequent". Rules are commonly used in expressing knowledge and are easily understood by human. Rule learning is the process of inducing rules from a set of training examples. Classical algorithms in this field include AQ15 [32] and CN2 [6]. Previous works in rule learning using Evolutionary Computation mainly use GA $[19,13]$. There are two different approaches. In the Michigan approach $[20,2]$ each individual in the GA corresponds to a rule, while in the Pittsburgh approach [37, 38] it corresponds to a set of rules. The system REGAL [12] uses the Michigan approach and a distributed genetic algorithm to learn first-order logic concept descriptions. It uses a selection operator, called Universal Suffrage operator, to achieve the learning of multi-modal concepts. Another system GABIL [23] uses the Pittsburgh approach. It can adaptively allow or prohibit certain genetic operations for certain individuals. GIL [22] also uses the Pittsburgh's approach and utilizes 14 genetic operators. These operators perform generalization, specialization or other modifications to the individuals at the rule set level, the rule level and the condition level.

\section{Causality and Structure Analysis}

The Causality and Structure Analysis process induces a Bayesian Network from the data. The learning approach is based on Lam and Bacchus's work $[27,26]$ on employing the Minimum Description Length principle to evaluate a Bayesian Network. Evolutionary 
Programming is employed to optimize this metric in order to search for the best network structure.

\subsection{The MDL metric}

The Minimum Description Length (MDL) metric measures the total description length $D_{t}(B)$ of a network structure $B$. A better network has a smaller value on this metric. Let $N=\left\{N_{1}, \ldots, N_{n}\right\}$ denote the set of nodes in the network (and thus the set of variables, since each node represents a variable), and $\Pi_{N_{i}}$ denote the set of parents of node $N_{i}$. The total description length of a network is the sum of description lengths of each node.

$$
D_{t}(B)=\sum_{N_{i} \in N} D_{t}\left(N_{i}, \Pi_{N_{i}}\right)
$$

This length is based on two components, the network description length $D_{n}$ and the data description length $D_{d}$.

$$
D_{t}\left(N_{i}, \Pi_{N_{i}}\right)=D_{n}\left(N_{i}, \Pi_{N_{i}}\right)+D_{d}\left(N_{i}, \Pi_{N_{i}}\right)
$$

The formula for the network description length is

$$
D_{n}\left(N_{i} \Pi_{N_{i}}\right)=k_{i} \log _{2}(n)+d\left(s_{i}-1\right) \prod_{j \in \Pi_{N_{i}}} s_{j}
$$

where $k_{i}$ is the number of parents of variable $N_{i}, s_{i}$ is the number of values $N_{i}$ can take on, $s_{j}$ is the number of values a particular variable in $\Pi_{N_{i}}$ can take on, and $d$ is the number of bits required to store a numerical value. This is the description length for encoding the 
network structure. The first part in the addition is the length for encoding the parents, while the second part is the length for encoding the probability parameters. This length can measure the simplicity of the network.

The formula for the data description length is

$$
D_{d}\left(N_{i}, \Pi_{N_{i}}\right)=\sum_{N_{i}, \Pi_{N_{i}}} M\left(N_{i,} \Pi_{N_{i}}\right) \log _{2} \frac{M\left(\Pi_{N_{i}}\right)}{M\left(N_{i}, \Pi_{N_{i}}\right)}
$$

where $M($.$) is the number of cases that match a particular instantiation in the database.$ This is the description length for encoding the data. A Huffman code is used to encode the data using the probability measure defined by the network. This length can measure the accuracy of the network.

\subsection{Combining MDL and EP}

We combined the MDL metric and EP for Bayesian Network Learning [41, 28]. The flowchart in Figure 3 shows the process. Each individual represents a network structure, which is a directed acyclic graph (DAG). A set of individuals is randomly created to make up the initial population. Each graph is evaluated by the MDL metric described above. Then each individual produces a child by performing a number of mutations. The child is also evaluated by the MDL metric. The next generation of population is selected among the parents and children by tournaments. Each DAG $B$ is compared with $q$ other randomly selected DAGs. The tournament score of $B$ equals to the number of rivals that $B$ can win, that is, the number of DAGs among those selected that have higher MDL scores than $B$. In our setting, the value of $q$ is 5 . Half of these DAGs with the 


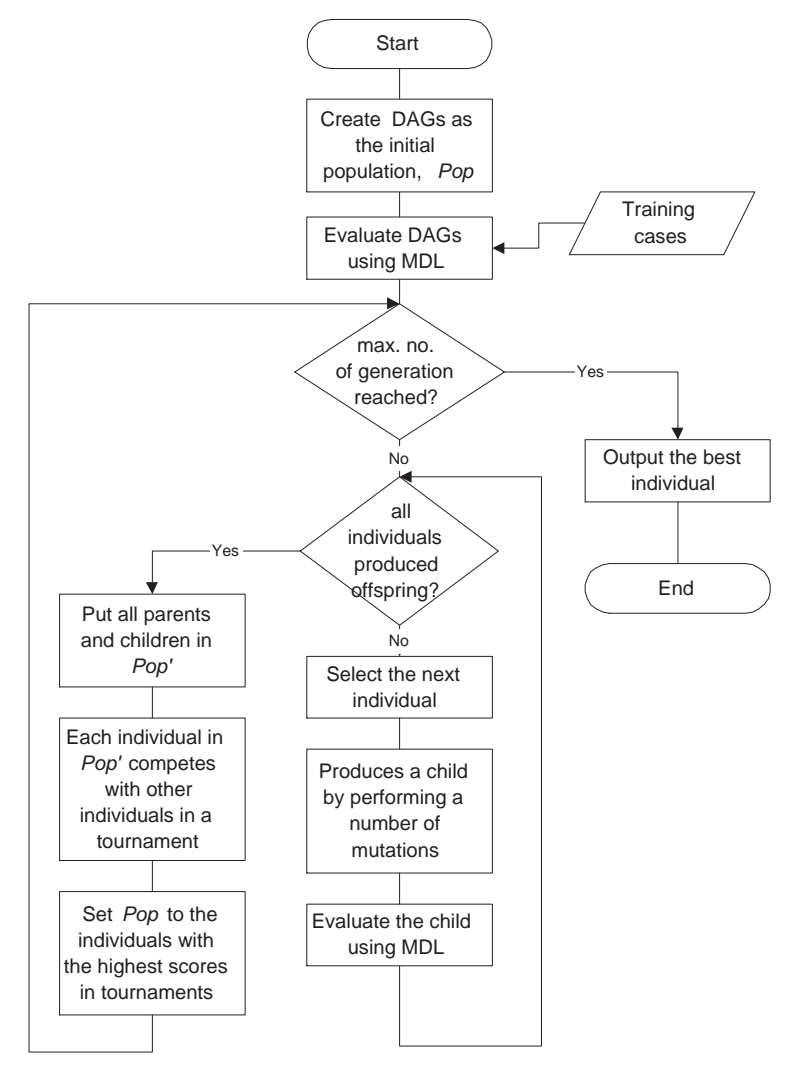

Figure 3: The flowchart of the Bayesian network learning process

higher tournament scores are retained as the next generation of population. The process is repeated until the maximum number of generations is reached. The setting on the maximum number of generations depends on the complexity of the network structure. If we expect a simple network, the maximum number of generations can be set to a lower value. The network with the lowest MDL score is output as the result.

\subsection{The Mutation Operators}

Offspring in EP is produced by using a number of mutations. The probabilities of using $1,2,3,4,5$ or 6 mutations are set to $0.2,0.2,0.2,0.2,0.1$ and 0.1 respectively. The mutation operators modify the edges of the DAG. If a cyclic graph is formed after the mutation, edges in the cycles are removed to keep it acyclic. Our approach uses four 
mutation operators, with the same probabilities of being used:

1. Simple mutation randomly adds an edge between two nodes or randomly deletes an existing edge from the parent.

2. Reversion mutation randomly selects an existing edge and reverses its direction.

3. Move mutation randomly selects an existing edge. It moves the parent of the edge to another node, or moves the child of the edge to another node.

4. Knowledge-Guided mutation is similar to simple mutation, but the MDL scores of the edges guide the selection of the edge to be added or removed. The MDL metric of all possible edges in the network is computed before the learning algorithm starts. This mutation operator stochastically adds an edge with a small MDL metric to the parental network or deletes an existing edge with a large MDL metric.

\section{Rule Learning}

The second step in our data mining process is to learn rules from the data. Our learning approach is based on Generic Genetic Programming (GGP) [43, 42, 40], which is an extension to Genetic Programming (GP). It uses a grammar [21] to control the structures evolved in GP.

\subsection{The Generic Genetic Programming Process}

The flowchart in Figure 4 shows the process of using GGP for rule learning. A grammar is provided as a template for rules. The algorithm starts with an initial population of 


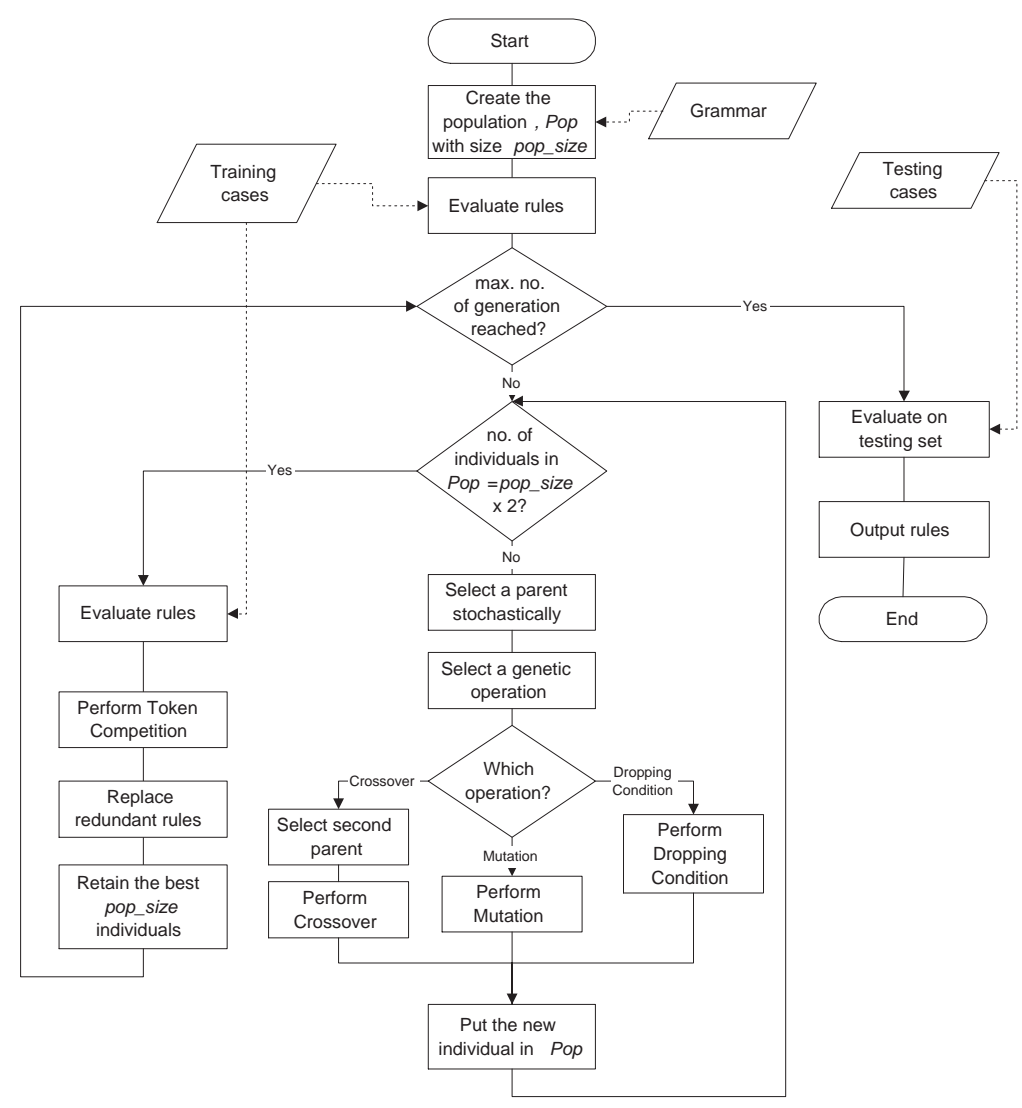

Figure 4: The flowchart of the Rule Learning process

randomly created rules using the grammar. One individual corresponds to one rule. Each rule is evaluated by a fitness function described in section 5.1. Then, individuals are selected stochastically to evolve offspring by the genetic operators. Rules with higher fitness scores have higher chances of being selected. The three genetic operators, crossover, mutation and dropping condition, are detailed in section 4.4. In each generation, the number of new individuals evolved equals to the population size. Thus at this stage, the number of individuals is doubled. All individuals participate in a token competition and a replacement step, so as to eliminate similar rules and increase the diversity. These two steps are presented in section 5.2. One half of the individuals with the higher fitness scores after token competition are passed to the next generation. 
To estimate the fitness scores of individuals a data set is used in GGP. The data set should be partitioned into a training set and a testing set. Only the training set is available for the learning process. After the maximum number of generations is reached, the discovered rules are further evaluated with the unseen testing set, so as to verify their accuracy and reject the rules that over-fit the training set. Our system uses $60 \%$ of the data as the training set and $40 \%$ as the testing set.

\subsection{Grammar}

The grammar governs the structures to be evolved from GGP. The format of rules in each problem can be different. Thus for each problem, a specific grammar is written so that the format of the rules can best fit the domain. The grammar should specify the structure of a rule. In general, the grammar specifies a rule is of the form "if antecedents then consequent". The antecedent part is a conjunction of attribute descriptors. The consequent part is an attribute descriptor as well. An attribute descriptor assigns a value to a nominal attribute, a range of values to a continuous attribute, or can be used to compare attribute values.

For example, consider a database with 4 attributes. We want to learn rules about attr4, which is Boolean. The attribute attr1 is nominal and coded with 0,1 or 2 . The attribute attr 2 is continuous between $0-200$. The domain of attr 3 is similar to attr2 and we want the rule to compare them.

An example of the context free grammar for rule learning in given in Table 1. The italic symbols are the non-terminals and the symbols with normal fonts are the terminals. A production rule of the form $\alpha \rightarrow \beta$ specifies that the non-terminals $\alpha$ can be expanded 


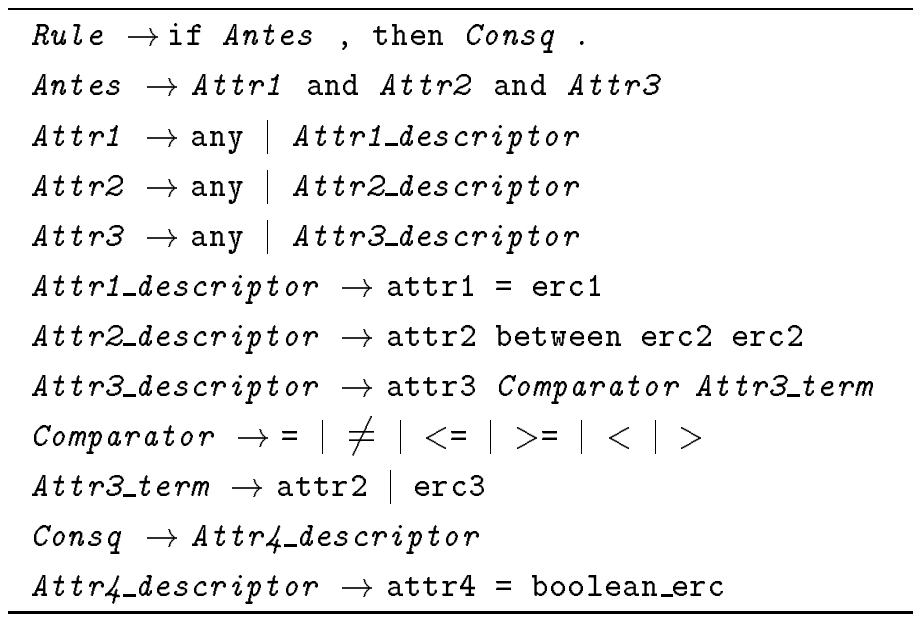

Table 1: An example grammar for rule learning.

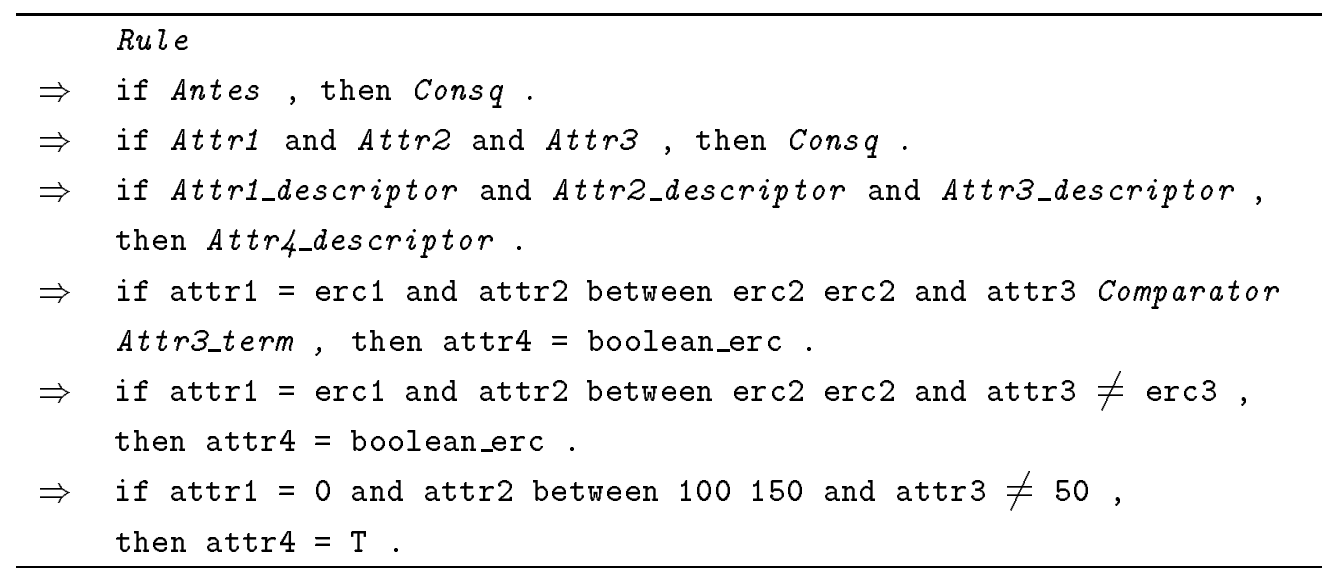

Table 2: An example derivation

to $\beta . \alpha \rightarrow \beta \mid \gamma$ is a short hand of $\{\alpha \rightarrow \beta, \alpha \rightarrow \gamma\}$. The symbols erc1, erc2, erc3 and boolean_erc in this grammar are ephemeral random constants (ERCs). Each ERC has it own range for instantiation: erc1 is within $\{0,1,2\}$, erc2 and erc3 is between $0-200$, boolean_erc can only be $\mathrm{T}$ or F. The symbol 'any' serves as a wild-card in the rule. An attribute will not be considered in the rule if its attribute descriptor is 'any'.

The grammar is used to derive rules to make up the initial population. The start symbol is the first symbol of the first line of the grammar. From the start symbol, a complete derivation is performed. Table 2 is an example of how a rule is derived from the 
grammar. This grammar allows rules like:

- if $\operatorname{attr} 1=0$ and attr 2 between 100150 and $\operatorname{attr} 3 \neq 50$, then attr $4=\mathrm{T}$.

- if $\operatorname{attr} 1=1$ and any and $\operatorname{attr} 3>=\operatorname{attr} 2$, then $\operatorname{attr} 4=\mathrm{F}$.

The grammars for other problems are similar to the grammar in Table 1. According to the type of the attribute, a descriptor similar to Attr1_descriptor, Attr2_descriptor or Attr3_descriptor can be used. GGP provides a powerful knowledge representation and allows a great flexibility on the rule format. The representation of rules is not fixed but depends on the grammar. The descriptor is not restricted to compare attributes with values. Rather, the descriptors can be comparisons between attributes. Rules with other formats can be learned, provided that the suitable grammar is supplied. Moreover, rules with the user desired structure can be learned because the user can specify the required rule format in the grammar.

\subsection{Use of Causality model and Temporal Order}

The use of grammar can ensure syntactical correctness in the rule, but not semantical correctness. It is desirable to eliminate meaningless rules in the search process. This requires a certain degree of knowledge on the causality between the attributes. The Causality and Structure analysis steps in our data mining module can provide this knowledge. The Bayesian network may provide an overview of the relationships among the attributes. For example, if we know that attribute $A$ is not related to any other attributes, then we don't need to learn rules about $A$. If we know attribute $B$ should depend on attributes $C$ and 
$D$, then we can specify a rule format like if $<$ attribute $C$ descriptor $>$ and $<$ attribute $D$ descriptor $>$, then <attribute $B$ descriptor $>$ '.

The temporal order among attributes can also provide knowledge to increase the learning efficiency. For example, in a medical domain, the rule "if treatment is plaster, then diagnosis is Radius fracture" is inappropriate. This rule does not make sense, because an operation is taken based on the treatment, not the other way round. In general, an event that occurs later will not be a cause of an event occurred earlier! Thus, we can order the attributes according the temporal relationship. The grammar should be designed such that an attribute is not placed in the 'if' part if it occurs later then the attribute in the 'then' part. This temporal order can be represented easily using a grammar. Both causality model and temporal order may significantly reduce search space and prune meaningless rules.

\subsection{Genetic Operators}

The search space is explored by generating new rules using three genetic operators: crossover, mutation and a newly defined operator called dropping condition. A rule is composed of attribute descriptors. The genetic operators try to change the descriptors in order to search for better rules. Rank selection [13] method is being used to select the parents. The probabilities of using crossover, mutation and dropping condition in our system are $0.5,0.4$ and 0.1 respectively.

Crossover is a sexual operation that produces one child from two parents. One parent is designated as the primary parent and the other one as the secondary parent. A part of the primary parent is selected and replaced by another part from the secondary parent. 
Suppose that the following primary and secondary parents are selected:

if $\operatorname{attr} 1=0$ and attr 2 between 100150 and $\operatorname{attr} 3 \neq 50$, then $\operatorname{attr} 4=\mathrm{T}$.

if $\operatorname{attr} 1=1$ and any and $\underline{\operatorname{attr} 3>=\operatorname{attr} 2}$, then $\operatorname{attr} 4=F$.

The underlined parts are selected for crossover. The offspring will be

if $\operatorname{attr} 1=0$ and $\operatorname{attr} 2$ between 100150 and $\underline{\operatorname{attr} 3>=\operatorname{attr} 2}$, then $\operatorname{attr} 4=\mathrm{T}$.

The replaced part is selected randomly from the primary parent, hence genetic changes may occur either on the whole rule, on several descriptors, or on just one descriptor. The replacing part is also selected randomly, but under the constraint that the offspring produced must be valid according to the grammar. If a conjunction of descriptors is selected, it will be replaced by another conjunction of descriptors, but never by a single descriptor. If a descriptor is selected, then it can only be replaced by another descriptor of the same attribute. This can maintain the validity of the rule.

Mutation is an asexual operation. The genetic changes may occur on the whole rule, several descriptors, one descriptor, or the constants in the rule. A part in the parental rule is selected and replaced by a randomly generated part. The new part is generated by the same derivation mechanism using the same grammar. Similar to crossover, because the offspring have to be valid according to the grammar, a selected part can only mutate to another part with a compatible structure. For example, the parent

if $\operatorname{attr} 1=0$ and $\operatorname{attr} 2$ between 100150 and $\operatorname{attr} 3 \neq 50$, then $\operatorname{attr} 4=\mathrm{T}$. may mutate to

if $\operatorname{attr} 1=0$ and attr 2 between 100150 and $\underline{\operatorname{attr} 3=40}$, then $\operatorname{attr} 4=\mathrm{T}$.

Due to the probabilistic nature of GP, redundant constraints may be generated in the rule. For example, suppose that the actual knowledge is 'if $A<20$ then $X=\mathrm{T}$ '. We 
may learn rules like 'if $A<20$ and $B<20$ then $X=\mathrm{T}$ '. This rule is, of course, correct; but it does not completely represent the actual knowledge. Dropping condition is an operator designed to generalize the rules. The rule can be generalized if one descriptor in the antecedent part is dropped. Dropping condition selects randomly one attribute descriptor, and then turns it into 'any'. That particular attribute is no longer considered in the rule, hence the rule can be generalized.

Reproduction operator is not used in our approach. In conventional GP, an individual can exploit its genetic material through the use of the reproduction operator. Good individuals can reproduce themselves in the population and gradually dominate the population. However, in our system, we do not want a good rule to replicate itself. Rather, we need to diversify the population in order to find several good rules. Hence reproduction is not used. Our system will only keep one copy for each good individual through token competition.

\section{$5 \quad$ Novel Techniques for Rule Learning}

Other than using GGP as the search algorithm, other techniques are needed so as to efficiently learn multiple interesting rules from the database. These techniques are described in this section.

\subsection{Evaluation of Rules}

Completeness and consistency are conventionally used as the evaluation metric. However, a complete rule covering all the database records is unrealistic in real-life situation. The 
support-confidence framework [1] is employed instead. Support measures the coverage of a rule. It is a ratio of the number of records covered by the rule to the total number of records. Confidence factor (cf) measures the consistency of a rule. It is the ratio of the number of records matching both the consequence and the antecedents to the number of records matching only the antecedents.

In the evaluation process, each rule is checked with every record in the training set. Three statistics are counted. The number antes_hit is the number of records matching the antecedents (the 'if' part), consq_hit is the number of records that match the consequent (the 'then' part), and both_hit is the number of records that obey the whole rule (both the 'if' and the 'then' parts).

The confidence factor $c f$ is the fraction both_hit/antes_hit. But a rule with a high confidence factor does not mean that it behaves significantly different from the average. Therefore we need to consider the average probability of consequent ( $p r o b$ ). The value prob is equal to consq_hit/total, where total is the total number of records in the training set. This value measures the confidence for the consequence under no particular antecedent.

We defined $c f_{-}$part as

$$
c f \_ \text {part }=c f \times \log \left(\frac{c f}{\text { prob }}\right)
$$

This value is based on two factors : $c f$ and $c f /$ prob. The log function measures the order of magnitude of the ratio $c f /$ prob. A high value of $c f$ _part requires the rule to have a high confidence $(c f)$ and $c f$ is higher than the average probability (prob).

Support is another measure that we need to consider. A rule can have a high accuracy but the rule may be just because of chance and based on a few training exam- 
ples. This kind of rules does not have enough support. The value of support is defined as both_hit/total. If support is below a user-defined minimum required support, min_support, the confidence factor of the rule should not be considered.

We define our fitness function to be:

$$
\text { raw_fitness }= \begin{cases}\text { support, } & \text { if support }<\text { min_support } \\ w_{1} \times \text { support }+w_{2} \times c f_{-p} \text { art }, & \text { otherwise }\end{cases}
$$

where the weights $w_{1}$ and $w_{2}$ are user-defined to control the balance between the confidence and the support. The values are set to 1 and 8 respectively so that the system prefers a rule with good confidence to a rule with good support.

\subsection{Token Competition}

One important requirement of a rule learning system is to learn as many interesting rules as possible. This can be modeled as the searching of multiple solutions in the search space. We follow the Michigan approach $[20,2]$ that each individual represents one rule. The individuals in the population combined together can provide a rule set. The token competition [31] technique is employed to achieve the niching [14] effect, so that good individuals in different niches are maintained in the population. Token competition has an advantage that it does not need to define and compare the similarity between individuals. It simply regards two individuals to be similar if they cover the same records.

In the natural environment, once an individual has found a good place for living, it will try to exploit this niche and prevent other newcomers to share the resources, unless the newcomer is stronger than it is. The other individuals are hence forced to explore and 
find their own niches. In this way, the diversity of the population is increased.

Based on this mechanism, we assume each record in the training set can provide a resource called token. If a rule can match a record, it will set a flag to indicate the token is seized. Other weaker rules then cannot get the token. The priority of receiving tokens is determined by the strength of the rules. A rule with a high score on raw_fitness can exploit the niche by seizing as many tokens as it can. The other rules entering the same niche will have their strength decreased because they cannot compete with the stronger rule. The fitness score of each individual is modified based on the token it can seize. The modified fitness is defined as :

$$
\text { modified_fitness }=\text { raw_fitness } \times \text { count } / \text { ideal }
$$

where count is the number of tokens that the rule actually seized, ideal is the ideal number of tokens that it can seize, which is equal to the number of records that the rule matches.

From another point of view, each rule contributes to the system by covering several records of the database. If a record has already been covered by one rule, then another rule covering the same record will make no contribution to the system. Thus the fitness of the latter rule should be discounted.

As a result of token competition, there are rules that cannot seize any token. These rules are redundant as all of its records are already covered by the stronger rules. They can be replaced by new individuals. Introducing these new individuals can inject a larger degree of diversity into the population, and provide extra chances for generating good rules. 


\begin{tabular}{||l|l|l|l||}
\hline \hline Name & Type & Description & Possible Value \\
\hline Sex & Nominal & Sex & 'M' or 'F' \\
\hline Age & Numeric & Age & Between 0 to 16 years old \\
\hline Admday & Date & Admission date & $\begin{array}{l}\text { Between year 1984 to 1996; Divided into four } \\
\text { parts: Day, Month, Year and Weekday }\end{array}$ \\
\hline Stay & Numeric & $\begin{array}{l}\text { Length of staying } \\
\text { in hospital }\end{array}$ & $\begin{array}{l}\text { Between 0 to 1000 days } \\
\text { Discretized into 18 non-uniform ranges. }\end{array}$ \\
\hline Diagnosis & Nominal & $\begin{array}{l}\text { Diagnosis of } \\
\text { fracture }\end{array}$ & $\begin{array}{l}\text { 10 different values, } \\
\text { based on the location of fracture }\end{array}$ \\
\hline Operation & Nominal & Operation & $\begin{array}{l}\text { 'CR' (Simple Closed Reduction), } \\
\text { 'CR+K-wire' (Closed Reduction with K-wire), } \\
\text { 'CR+POP' (Closed Reduction with POP), } \\
\text { 'OR' (Open Reduction) or Null (no operation) }\end{array}$ \\
\hline Surgeon & Nominal & Surgeon & One of 61 surgeons or Null if no operation \\
\hline Side & Nominal & Side of fracture & 'Left', 'Right', 'Both' or 'Missing' \\
\hline \hline
\end{tabular}

Table 3: Attributes in the fracture database.

\section{Results on the Fracture Database}

The described data mining technology has been applied to a real-life medical database consisting of children with limb fractures, admitted to the hospital in the period 19841996. These data can provide information for the analysis of children fracture patterns. This database has 6500 records and 8 attributes. The attributes are listed in Table 3 .

\subsection{Results of Causality and Structure Analysis}

The relationships among the attributes are analyzed by learning a Bayesian network. We have used a typical population size of 50 to run for 100 generations. The execution time is 45 minutes. The best network structure learned is drawn in Figure 5. Day, Month, Weekday and Year refer to different parts of the admission date.

This network shows three chains of causalities. The first chain shows that the length of staying in hospital depends on the operation, the operation in turn depends on the 


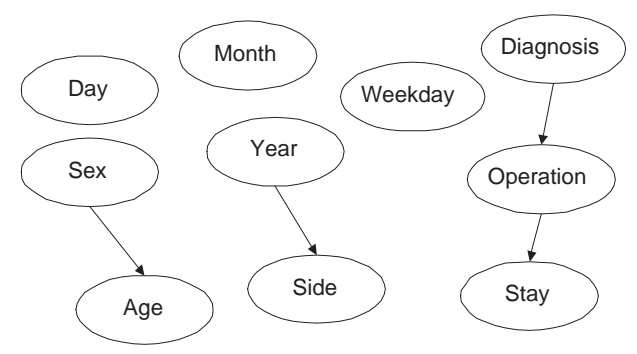

Figure 5: The best network structure for the fracture database

diagnosis. Another edge in the network is between Sex and Age. Although by common sense sex should not be the cause of age, in fact in the database the age is correlated with sex. Female patients are more likely to be in the younger age group (from age 0 to age 7 ) , and male patients are more likely to be in the elder age group (from 11 to 15 ). There is another edge between Year and Side. This result is quite surprising. The conditional probabilities are investigated and two interesting points are revealed.

- The probabilities for side equal to 'both' are exceptionally low for year 1984, 1988 and 1992.

- The probabilities for missing the attribute Side is high for year 1995 and 1996, while this probabilities for other years are low.

This phenomenon cannot be explained reasonably. We suspected that different notations are used in recording the side for different years.

\subsection{Results of Rule Learning}

Based on the learned Bayesian network, we observed a causality model between diagnosis, operation and stay. We wished to learn knowledge about these attributes. In addition, the temporal order gives extra knowledge on how the rules should be formulated. The 


\begin{tabular}{|c|c|c|c|c|c|c|c|c|c|c|}
\hline \multirow{2}{*}{ About } & No. of & \multicolumn{4}{|c|}{$c f$} & \multicolumn{3}{c|}{$c f /$ prob } & \multicolumn{3}{c|}{ support } \\
\cline { 3 - 12 } & Rules & mean & $\max$ & min & mean & $\max$ & $\min$ & mean & $\max$ & $\min$ \\
\hline Diagnosis & 2 & $45.6 \%$ & $51.4 \%$ & $39.8 \%$ & 1.6 & 1.7 & 1.4 & $9.2 \%$ & $10.0 \%$ & $8.4 \%$ \\
\hline Operation & 8 & $42.6 \%$ & $74.0 \%$ & $28.0 \%$ & 2.0 & 2.9 & 1.1 & $5.4 \%$ & $16.2 \%$ & $3.2 \%$ \\
\hline Stay & 7 & $71.1 \%$ & $81.1 \%$ & $47.0 \%$ & 2.5 & 7.0 & 1.4 & $4.5 \%$ & $8.7 \%$ & $3.1 \%$ \\
\hline
\end{tabular}

Table 4: Summary of the rules for the fracture database

attributes can be divided into three time stages: A diagnosis is first given to the patient, then an operation is taken, and after that the patient stays in the hospital. This knowledge leads to three causality models. Firstly, sex, age and admission date are the possible causes of diagnosis. Secondly, these three attributes and diagnosis are the possible causes of operation and surgeon. Thirdly, length of staying has the other attributes as the possible causes. A grammar (see Appendix A) is written as a template for these three kinds of rules. We have used a population size of 300 to run for 50 generations in the rule learning step. The execution time is about 3 hours for the 6500 records. The results are listed in Table 4.

Two interesting rules about diagnosis are found. An example is:

If age is between 2 and 5 , then diagnosis is Humerus. ( $c f=51.43 \%)$

The confidences of the rules are just around 40\%-50\%. It is partly because there are actually no strong rules affecting the value of diagnosis. However the ratio $c f /$ prob shows that the patterns discovered deviated significantly from the average. We found that humerus fracture is the most common fracture for children between 2 and 5 years old. Radius fracture is the most common fracture for boys between 11 and 13 .

Eight interesting rules about operation are found. An example is:

If age is between 0 and 7, and admission year is between 1988 and 1993, and diagnosis is Radius, then operation is $\mathrm{CR}+\mathrm{POP}$. ( $c f=74.05 \%)$ 
These rules suggest that radius and ulna fractures are usually treated with CR+POP (i.e. plaster). Operation is usually not needed for tibia fracture. Open reductions are more common for elder children with age larger than 11, while young children with age lower than 7 have a higher chance of not needing operations. We did not find any interesting rules about surgeons, as the surgeons for operation are more or less randomly distributed in the database.

Seven interesting rules about length of staying are found. An example is: If admission year is between 1985 and 1996, and diagnosis is Femur, then stay is more than 8 days. ( $f=81.11 \%)$

The results suggest that Femur and Tibia fractures are serious injuries and have to stay longer in hospital. If open reduction is used, the patient requires longer time to recover because the wound has been cut open for operation. If no operation is needed, it is likely that the patient can return home within one day. Relatively, radius fracture requires a shorter time for recovery.

The results have been evaluated by the medical experts. The causality model matches with the general knowledge. The doctor decides a treatment based on the type of fracture, and the treatment affects the recovery. Previous analyses on fracture patterns only gave an overall injury pattern. Our system automatically uncovered relationships between different attribute values. The rules provide interesting patterns that were not recognized before. The analysis gives an overview of the important epidemiological and demographic data of the fractures in children. It clearly demonstrated the treatment pattern and rules of decision making. It can provide a good monitor of the change of pattern of management and the epidemiology if the data mining process is continued longitudinally 


\begin{tabular}{||l|l|l||}
\hline \hline Name & Explanation & Possible Value \\
\hline Sex & Sex & 'M' or 'F' \\
\hline Age & Age & positive integer \\
\hline Lax & Joint Laxity & integer betweeen 0 and 3 \\
\hline 1stCurveT1 & Whether 1st curve started at vertebra T1 & Y or N \\
\hline 1stMCGreater & $\begin{array}{l}\text { Whether the degree of 1st Major Curve } \\
\text { is greater the the 2nd Major Curve }\end{array}$ & Y or N \\
\hline L4Tilt & Whether vertebra L4 is tilted & Y or N \\
\hline 1stMCDeg & Degree of 1st Major Curve & positive integer \\
\hline 2ndtMCDeg & Degree of 2nd Major Curve & positive integer \\
\hline 1stMCApex & Apex of 1st Major Curve & any vertebra \\
\hline 2ndMCApex & Apex of 2nd Major Curve & any vertebra \\
\hline Deg1 & Degree of 1st Curve & positive integer \\
\hline Deg2 & Degree of 2nd Curve & positive integer \\
\hline Deg3 & Degree of 3rd Curve & positive integer \\
\hline Deg4 & Degree of 4th Curve & positive integer \\
\hline Class & Scoliosis Classification & K-I, K-II, K-III, K-V, TL, L \\
\hline Mens & Period of Menstruation & positive integer \\
\hline TSI & Trunk Shift (in cm) & positive integer \\
\hline TSIDir & Trunk Shift Direction & null, left or right \\
\hline RI & Risser Sign & integer between 0 and 5 \\
\hline Treatment & Treatment & Observation, surgery or bracing \\
\hline \hline
\end{tabular}

(Vertebras are coded with T1-T12 or L1-L5)

(Trunk Shift measures the displacement of the curve)

(Risser Sign measures the maturity of the patient)

Table 5: Attributes in the Scoliosis database

over the years. It also helps to provide the information for setting up a knowledge-based instruction system to help young doctors in training to learn the rules in diagnosis and treatment.

\section{Results on the Scoliosis Database}

The data mining process has been applied to another database. The second database con-

tains clinical records of patients with Scoliosis. Scoliosis refers to the spinal deformation.

A Scoliosis patient has one or several curves in his spine. Among them, the curves with 


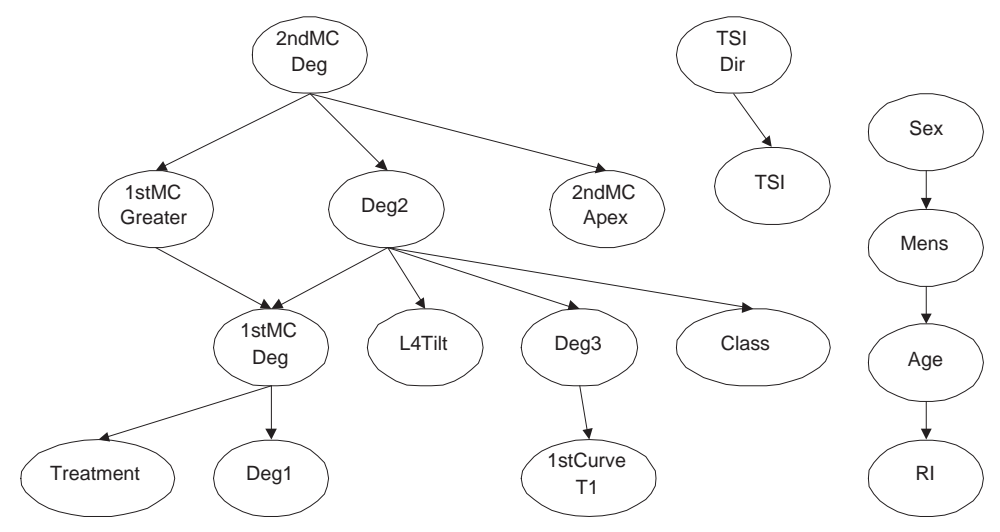

Figure 6: The best network structure for the Scoliosis database

severe deformations are identified as major curves. The database stores measurements on the patients, such as the number of curves, the curve locations, degrees and directions. It also records the maturity of the patient, the class of Scoliosis and the treatment. The database has 500 records. According to the domain expert, 19 attributes are useful and extracted from the database in the preprocessing step. They are shown in Table 5.

\subsection{Results of Causality and Structure Analysis}

We have used a population size of 50 and a maximum number of generations of 1000 to run in the causality and structure analysis. The execution time is 3 minutes. The best Bayesian network structure learned is drawn in Figure 6. The right part of the network shows that sex implies menstruation, and menstruation implies age, and age in turn implies RI. The network also shows that TSIDir can imply TSI because if TSI direction is null, TSI should be 0 .

The main part of the network shows that 2ndMCDeg can imply 2ndMCApex and 1stMCGreater. This is because if 2 ndMCDeg $=0$, the patient does not have the second major curve, and thus 2ndMCApex must be null and the first major curve must be the greater curve. The 
value of 2ndMCDeg also imply the degree of the second curve (Deg2), because if the patient has two major curves, most of the time the second major curve is the second curve. The value of 1stMCDeg is affected by 1stMCGreater and Deg2. When the degree of first major curve is greater than the second curve, most likely 1stMCDeg is large. When Deg2 is large, most likely the first major curve will be the second curve. The value of 1stMCDeg can imply Deg1 because when the value of 1stMCDeg is small, the degree first curve is not large. Deg2 can imply the value of L4Tilt and Deg3, while Deg3 can imply 1stCurveT1. If degree of the second curve is large, then usually L4 is tilt. If the patient does not have the second curve, then he will not have the third curve. Moreover, if he has at least three curves, then most of the time the deformation will start at the first vertebra T1. The network also shows that the value of treatment mainly depends on 1stMCDeg. On the other hand, Class depends on Deg2.

\subsection{Results of Rule Learning}

The medical experts are interested to discover knowledge about classification of Scoliosis and treatment. Scoliosis can be classified as Kings, Thoracolumbar(TL) and Lumbar(L), while Kings can be further subdivided into K-I, II, III, IV and V. Treatment can be observation, surgery and bracing. The determinations of these two attributes are complicated. The rule learning step can induce rules about them. Although the induced Bayesian network provides valid and useful relationships, the domain expert is more interested in finding relationships between classification and the attributes 1stCurveT1, 1stMCGreater, L4Tilt, 1stMCDeg, 2ndMCDeg, 1stMCApex and 2ndMCApex, and relationships between treatment and age, laxity, degrees of the curves, maturity of the patient, 


\begin{tabular}{|c|c|c|c|c|c|c|c|c|}
\hline \multirow{2}{*}{ Class } & No. of & \multicolumn{3}{|c|}{$c f$} & \multicolumn{3}{|c|}{ support } & \multirow{2}{*}{ prob } \\
\cline { 3 - 7 } & Rules & mean & max & min & mean & max & min & \\
\hline King-I & 5 & $94.84 \%$ & $100 \%$ & $90.48 \%$ & $5.67 \%$ & $10.73 \%$ & $0.86 \%$ & $28.33 \%$ \\
\hline King-II & 5 & $80.93 \%$ & $100 \%$ & $52.17 \%$ & $6.61 \%$ & $14.38 \%$ & $1.07 \%$ & $35.41 \%$ \\
\hline King-III & 4 & $23.58 \%$ & $25.87 \%$ & $16.90 \%$ & $1.56 \%$ & $2.58 \%$ & $0.86 \%$ & $7.94 \%$ \\
\hline King-IV & 2 & $24.38 \%$ & $29.41 \%$ & $19.35 \%$ & $1.18 \%$ & $1.29 \%$ & $1.07 \%$ & $2.79 \%$ \\
\hline King-V & 5 & $54.13 \%$ & $62.50 \%$ & $45.45 \%$ & $0.97 \%$ & $1.07 \%$ & $0.86 \%$ & $6.44 \%$ \\
\hline TL & 1 & $41.18 \%$ & $41.18 \%$ & $41.18 \%$ & $1.50 \%$ & $1.50 \%$ & $1.50 \%$ & $2.15 \%$ \\
\hline L & 3 & $54.04 \%$ & $62.50 \%$ & $45.45 \%$ & $2.00 \%$ & $2.79 \%$ & $1.07 \%$ & $4.51 \%$ \\
\hline
\end{tabular}

Table 6: Results of the rules for Scoliosis classification

displacement of the vertebra and the class of Scoliosis. This domain knowledge can be easily incorporated in the design of the rule grammar. There are two types of rules, one for classification of Scoliosis and the other for suggesting treatment. The grammar is outlined in Appendix B.

The population size used in the rule learning step is 100 and the maximum number of generations is 50. The execution time is about 1 hour. The results of rule learning from this database are listed below.

1. Rules for Scoliosis classification.

An example of this kind of rules is like:

if 1 stMCGreater $=\mathrm{N}$ and 1stMCApex $=\mathrm{T} 1-\mathrm{T} 8$ and 2ndMCApex $=$ L3-L4, then King-I. $(\mathrm{cf}=100 \%)$

For each class of Scoliosis, a number of rules are mined. The results are summarized in Table 6 . These results have been compared with the knowledge of doctors. For King-I and II, the rules have high confidence and generally match with the knowledge of medical experts. However there is one unexpected rule for the classification of King-II. Under the conditions specified in the antecedents, our system found a 
confidence factor of $52 \%$ for classifying to King-II. However, the domain expert suggests the class should be King-V! After an analysis on the database, we revealed that serious data errors existed in the current database. Some records contain incorrect Scoliosis classification.

For King-III and IV, the confidence of the rules discovered is just around $20 \%$. According to the domain expert, one common characteristic for these two classes is that there is only one major curve or the second major curve is insignificant. However there is no rigid definition for a 'major curve' and the concept of 'insignificant' is fuzzy. These depend on the interpretation of doctors. Because of the lack of this important information, the system cannot find accurate rules for these two classes. Another problem is that King-III or IV only occupied a small proportion of the database, as shown by prob in Table 6 . The database cannot provide a large number of cases for training. Similar problems also existed for King-V, TL and L.

For the class King-V, TL and L, the system found rules with confidence around $40 \%$ to $60 \%$. Nevertheless, the rules for TL and L show something different in comparison with the existing domain knowledge. According to our rules, the classification always depends on the first major curve, while according to the domain expert, the classification depends on the larger major curve. After discussion with the domain expert, it is agreed that our rules are more accurate than the existing domain knowledge.

2. Rules about treatment.

An typical rule of this kind is: 


\begin{tabular}{|c|c|c|c|c|c|c|c|c|}
\hline Type & No. of & \multicolumn{3}{|c|}{$c f$} & \multicolumn{3}{|c|}{ support } & \multirow{2}{*}{ prob } \\
\cline { 3 - 8 } & Rules & mean & $\max$ & $\min$ & $\operatorname{mean}$ & $\max$ & $\min$ & \\
\hline Observation & 4 & $98.89 \%$ & $100 \%$ & $95.55 \%$ & $3.49 \%$ & $6.01 \%$ & $1.07 \%$ & $62.45 \%$ \\
\hline Bracing & 5 & $79.57 \%$ & $100 \%$ & $71.43 \%$ & $1.03 \%$ & $1.29 \%$ & $0.86 \%$ & $24.46 \%$ \\
\hline Surgery & 0 & - & - & - & - & - & - & $3.65 \%$ \\
\hline
\end{tabular}

Table 7: Results of the rules about treatment

If age=2-12 and Deg1=20-26 and Deg2=24-47 and Deg3=27-52 and Deg4=0, then Bracing. ( $c f=100 \%)$

The results are summarized in Table 7 . The rules for observation and bracing have very high confidence factors. However, the support is not high that the rules only cover fragments of the cases. Our system prefers accurate rules to general rules. If the user prefers more general rules, the weights in the fitness function can be tuned. For surgery, no interesting rule was found because only $3.65 \%$ of the patients are treated with surgery.

The biggest impact on the clinician from the data mining analysis of the Scoliosis database is the fact that many rules set out in the clinical practice are not clearly defined. The usual clinical interpretation depends on the subjective experience of the clinician. Data mining revealed quite a number of mismatches in the classification on the type of Kings curves. After a careful review by the senior surgeon it appears that the database entry by many junior surgeons are not accurate and that the data mining rules are in fact more accurate! This shows that the rules must be more clearly defined quantitatively and that data mining rules can help in the training of younger doctors and act as an intelligent means to validate and evaluate the accuracy of the clinical database. An accurate and validated clinical database is very important for helping clinicians to make decisions, to 
assess and evaluate treatment strategies, to conduct clinical and related basic research, to enhance teaching and professional training.

\section{Conclusion}

We have presented a data mining system that is composed of five steps. The third and fourth steps are detailed. They both employ Evolutionary Computation as the search algorithms. Causality and Structure Analysis focuses on the general causality model between the variables while Rule Learning captures the specific behavior between particular values of the variables.

Our system is particular suitable to real-life databases that cannot be described completely by just a few rules. Building a complete model for such a database is difficult and usually results in a complicated model. We have used a Bayesian network to give a causality model. The Bayesian network is easy to understand while it has a well-developed mathematical model. Moreover, in many real-life situation, the rules existed are just general guidelines with many exceptional cases. The rule learning step aims to learn such kind of knowledge. It compares the confidence of the rule with the average probability and search for the patterns significantly deviated from the normal. Token competition is used so as to learn as many rules as possible. Furthermore, knowledge from domain experts can be very useful to data mining. The use of grammar allows the domain knowledge to be easily and effectively utilized. The grammar on one hand can prune the search space on meaningless rules while on the other hand can ensure that the output knowledge is in the user desired format. 
The system has been applied to two real-life medical databases. The results can provide interesting knowledge as well as suggest refinements to the existing knowledge. We even found unexpected results that lead to discovery of errors in the database. In the fracture database, the system automatically uncovered knowledge about the age effect on fracture, the relationship between diagnoses and operations, and the effect of diagnoses and operations on lengths of staying in the hospital. In the Scoliosis database, we have discovered new knowledge about the classification of Scoliosis and about the treatment. The knowledge leads to refinements of the existing knowledge.

\section{Acknowledgments}

The work was partially supported by Hong Kong RGC CERG Grant CUHK 4161/97E. The authors wish to thank Ms. Chun Sau Lau and Ms. King Sau Lee for preparing, analyzing and implementing the rule learning system for the Scoliosis database.

\section{References}

[1] R. Agrawal, T. Imielinski, and A. Swami. Mining association rules between sets of items in large databases. In Proceedings of the 1993 International Conference on Management of Data (SIGMOD 93), pages 207-216, 1993.

[2] L. Booker, D. E. Goldberg, and J. H. Holland. Classifier systems and genetic algorithms. Artificial Intelligence, 40:235-282, 1989. 
[3] R. R. Bouckaert. Properties of belief networks learning algorithms. In Proceedings of the Conference on Uncertainty in Artificial Intelligence, pages 102-109, 1994.

[4] E. Charniak. Bayesian networks without tears. AI Magazine, 12(4):50-63, 1991.

[5] C. K. Chow and C. N. Liu. Approximating discrete probability distributions with dependence trees. IEEE Transactions on Information Theory, 14(3):462-467, 1968.

[6] P. Clark and T. Niblett. The CN2 induction algorithm. Machine Learning, 3:261-283, 1989.

[7] G. F. Cooper. The computational complexity of probabilistic inference using Bayesian belief networks. Artificial Intelligence, 42:393-405, 1990.

[8] G. F. Cooper and E. Herskovits. A Bayesian method for the induction of probabilistic networks from data. Machine Learning, 9:309-347, 1992.

[9] U. M. Fayyad, G. Piatesky-Shapiro, and P. Smyth. From data mining to knowledge discovery : An overview. AI Magazine, pages 37-51, Fall 1996.

[10] D. B. Fogel. An introduction to simulated evolutionary optimization. IEEE Transactions on Neural Network, 5:3-14, 1994.

[11] L. Fogel, A. Owens, and M. Walsh. Artificial Intelligence through Simulated Evolution. New York: John Wiley and Sons, 1966.

[12] A. Giordana and F. Neri. Search-intensive concept induction. Evolutionary Computation, 3:375-416, 1995 . 
[13] D. E. Goldberg. Genetic Algorithms in Search, Optimization and Machine Learning. Addison-Wesley, 1989.

[14] D. E. Goldberg and J. Richardson. Genetic algorithms with sharing for multimodal function optimization. In Proceedings of the second International Conference on Genetic Algorithms, pages 41-49, 1987.

[15] D. Heckerman. Bayesian Networks for Knowledge Discovery, chapter 11, pages 273306. AAAI/MIT Press, 1996.

[16] D. Heckerman, D. Geiger, and D. M. Chickering. Learning Bayesian networks: The combination of knowledge and statistical data. Machine Learning, 20(3):197-243, 1995.

[17] D. Heckerman and M. P. Wellman. Bayesian networks. Communications of the ACM, 38(3):27-30, March 1995.

[18] E. Herskovits and G. Cooper. KUTATO: An entropy-driven system for construction of probabilistic expert systems from databases. Technical Report KSL-90-22, Knowledge Systems Laboratory, Medical Computer Science, Stanford University, 1990.

[19] J. H. Holland. Adaptation in Natural and Artificial Systems. Bradford/MIT Press, 1992.

[20] J. H. Holland and J. S. Reitman. Cognitive systems based on adaptive algorithms. In D. A. Waterman and F. Hayes-Roth, editors, Pattern-Directed Inference Systems. Academic Press, 1978. 
[21] J. E. Hopcroft and J. D. Ullman. Introduction to automata theory, languages, and computation. Reading, MA: Addison-Wesley, 1979.

[22] C. Z. Janikow. A knowledge-intensive genetic algorithm for supervised learning. Machine Learning, 13:189-228, 1993.

[23] K. A. De Jong, W. M. Spaers, and D. F. Gordon. Using genetic algorithms for concept learning. Machine Learning, 13:161-188, 1993.

[24] J. R. Koza. Genetic Programming : on the programming of computers by means of natural selection. Bradford/MIT Press, 1992.

[25] J. R. Koza. Genetic Programming II : automatic discovery of reusable programs. Bradford/MIT Press, 1994.

[26] W. Lam. Bayesian network refinement via machine learning approach. IEEE Transactions on Pattern Analysis and Machine Intelligence, 1998.

[27] W. Lam and F. Bacchus. Learning Bayesian belief networks - an approach based on the MDL principle. Computational Intelligence, 10(3):269-293, 1994.

[28] W. Lam, M. L. Wong, K. S. Leung, and P. S. Ngan. Discovering probabilistic knowledge from databases using evolutionary computation and minimum description length principle. In Genetic Programming 1998: Proceedings of the Third Annual Conference, 1998.

[29] P. Larranaga, M. Poza, Y. Yurramendi, R. Murga, and C. Kuijpers. Structure learning of Bayesian network by genetic algorithms: A performance analysis of control pa- 
rameters. IEEE Transactions on Pattern Analysis and Machine Intelligence, 18(9):9, 1996.

[30] P. Larranage, C. Kuijpers, R. Murga, and Y. Yurramendi. Learning Bayesian network structures by searching for the best ordering with genetic algorithms. IEEE Transactions on System, Man, and Cybernetics - Part A: Systems and Humans, 26(4):487-493, 1996 .

[31] K. S. Leung, Y. Leung, L. So, and K. F. Yam. Rule learning in expert systems using genetic algorithm: 1, concepts. In Proceedings of the 2nd International Conference on Fuzzy Logic and Neural Networks(Iizuka, Japan), pages 201-204, 1992.

[32] R. S. Michalski, I. Mozetic, J. Hong, and N. Lavrac. The multi-purpose incremental learning system AQ15 and its testing application to three medical domains. In Proceedings of the 5th National Conference on Artificial Intelligence, pages 1041-1045, 1986.

[33] G. Rebane and J. Pearl. The recovery of causal poly-trees from statistical data. In Uncertainty in Artificial Intelligence 3, pages 175-182. North-Holland, Amsterdam, 1989.

[34] I. Rechenberg. Evolution Strategy: Optimization of technical systems by means of biological evolution. Stuttgart: Fromman-Holzboog, 1973.

[35] H. P. Schwefel. Numerical Optimization of Computer Models. Chichester: Wiley, 1981. 
[36] M. Singh and M. Valtorta. An algorithm for the construction of Bayesian network structures from data. In Proceedings of the Conference on Uncertainty in Artificial Intelligence, pages 259-265, 1993.

[37] S. F. Smith. A Learning System based on Genetic Adaptive Algorithms. PhD thesis, University of Pittsburgh, 1980.

[38] S. F. Smith. Flexible learning of problem solving heuristics through adaptive search. In Proceedings of the Eighth International Conference On Artificial Intelligence. Morgan Kaufmann, 1983.

[39] P. Spirtes, C. Glymour, and R. Scheines. Causation, Prediction and Search. SpringerVerlag, 1993.

[40] M. L. Wong. Evolutionary program induction directed by logic grammars. PhD thesis, The Chinese University of Hong Kong, 1995.

[41] M. L. Wong, W. Lam, and K. S. Leung. Using evolutionary computation and minimum description length principle for data mining of probabilistic knowledge. submitted to IEEE Transactions on Pattern Analysis and Machine Intelligence, 1997.

[42] M. L. Wong and K. S. Leung. Inducing logic programs with genetic algorithms: The genetic logic programming system. IEEE Expert, 10(5):68-76, 1995.

[43] M. L. Wong and K. S. Leung. Evolutionary program induction directed by logic grammars. Evolutionary Computation, 5:143-180, 1997. 


\section{Appendices}

\section{A The grammar for the fracture database}

This grammar is not completely listed. The grammar for the other attribute descriptors is similar to the part of the grammar in lines 11-19.

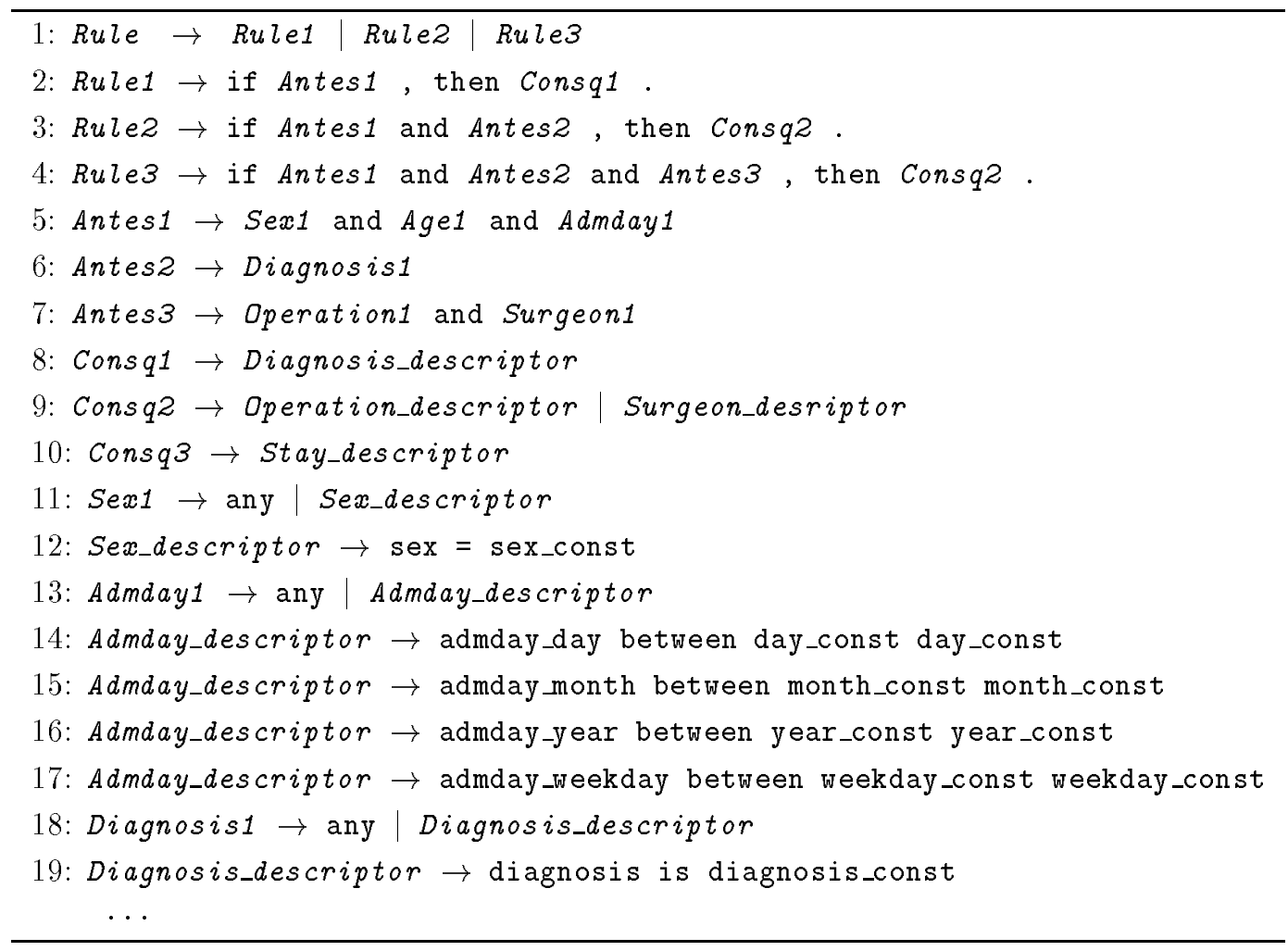

\section{B The grammar for the Scoliosis database}

This grammar is not completely listed. The grammar for the other attribute descriptors is similar to the part of the grammar in lines 7-12. 


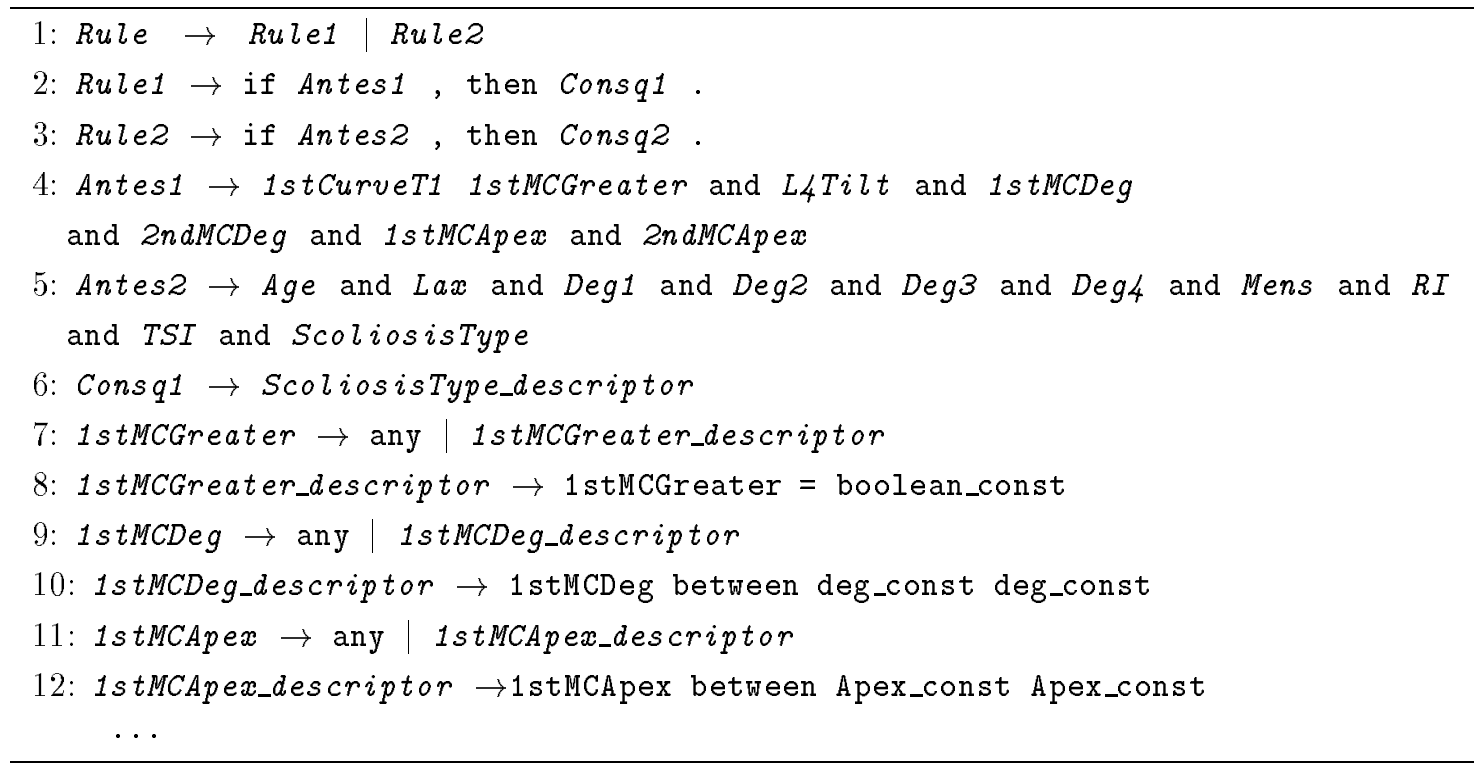

